\section{Erstunterzeichner:}

Prof.Dr. Rolf-Peter Calliess, Hannover; Prof.Dr.Heinz Cornel, Berlin; Prof.Dr. Frieder Dünkel, Greifswald; Prof.Dr. Bernd Maelicke, Lüneburg; Prof.Dr.Dr.h.c. Heinz Müller-Dietz, Sulzburg; Prof.Dr. Horst Schüler-Springorum, München; Prof.Dr. Bernd-Rüdeger Sonnen, Hamburg; Prof.Dr. Michael Walter, Köln

\section{weitere Unterzeichner:}

Dr.Kai Bammann, Bremen; Prof.Dr. Britta Bannenberg, Bielefeld; Dr. Michael C. Baurmann, Mainz; Dr. Mechthild Bereswill, Hannover; Prof.Dr. Werner Beulke, Passau; Prof.Dr. Lorenz Böllinger; Prof.Dr. Klaus Boers, Münster; Dr. Axel Boetticher, Karlsruhe; Oliver Brüchert, Frankfurt am Main; Dr. Burkhard Damman, Wien; Prof.Dr. Dieter Dölling, Heidelberg; Prof.Dr. Marlis Dürkop, Hamburg; Prof.Dr. Helga Einsele, Frankfurt am Main; Prof.Dr. Ulrich Eisenberg, Berlin; Prof.Dr. Johannes Feest, Bremen; Prof.Dr. Thomas Feltes, Bochum; Prof.Dr. Monika Frommel, Kiel; Prof.Dr. Brigitte Geissler-Piltz, Berlin; Jochen Goerdeler, Hannover; Achim Golzem, Frankfurt; Prof.Dr. Ute Ingrid Haas, Wolfenbüttel; Gernot Hahn, Fürth; Christoph Freiherr von Harsdorf, Wien; Prof.Dr .Arthur Hartmann, Bremen; Prof.Dr. Wolfgang Heinz, Konstanz; Dr. Olaf Heischel, Berlin; Prof.Dr. Peter Höflich, Cottbus; Prof.Dr. Frank Höpfel, Wien; Prof. Konrad Huchting, Emden; Prof.Dr. Herbert Jäger, Hamburg; Prof.Dr. Udo Jesionek, Wien; Prof.Dr. Heike Jung, Saarbrücken; Prof.Dr. Günther Kaiser, Freiburg; Prof. Gabriele Kawamura-Reindl, Nürn- berg; Prof.Dr. Hans-Jürgen Kerner, Tübingen; Prof.Dr. Joachim Kersten, Villingen-Schwenningen; Dr.Jörg Kinzig, Freiburg; Prof.Dr. Gerd Ferdinand Kirchhoff, Mönchengladbach; Prof.Dr. Diethelm Klesczewski, Leipzig; Prof.Dr. Peter Knösel, Potsdam; Prof. Gertrud Krauss, Nürnberg; Prof.Dr. Arthur Kreuzer, Gießen; Prof.Dr. Hans-Ludwig Kröber; Prof.Dr. Timm Kunstreich, Hamburg; Dr. Liora Lazarus, Oxford; Dr. Werner Lehne, Hamburg; Prof.Dr. Michael Matzke, Berlin; Prof.Dr. Bernd Dieter Meier, Hannover; Prof.Dr. Norbert Nedopil, München; Dr. Frank Neubacher, Köln; Prof. Werner Nickolai, Freiburg; Prof.Dr. Gerhard Nothacker, Potsdam; Prof.Dr. Sabine Nowara, Waltrop; Prof.Dr. Heribert Ostendorf; Prof.Dr. Hans-Uwe Otto, Bielefeld; Dr. Werner Päckert, Taunusstein; Prof.Dr. Hans-Joachim Plewig, Lüneburg; Dr. Gerhard Rehn, Hamburg; Prof.Dr.Richard Reindl, Nürnberg; Prof.Dr. Klaus Riekenbrauk, Düsseldorf; Dr. Dorothea Rzepka, Frankfurt; Dr. Karl-Peter Rotthaus, Präsident des Justizvollzugsamtes Rheinland a.D., Schondorf; Prof.Dr. Albert Scherr, Freiburg; Prof.Dr.Heinz Schöch, München; Prof.Dr. Lorenz Schulz, Frankfurt; Prof.Dr. Klaus Sessar, Hamburg; Prof. Wolfhart Sommerlad, Frankfurt am Main; Prof.Dr.Heinz Steinert, Frankfurt am Main; Prof.Dr. Franz Streng, Erlangen; Prof.Dr. Andreas Strunk, Wernau; Prof.Dr. Thomas Trenczek, Jena; Prof.Dr. Bernhard Villmow, Hamburg; Prof.Dr. Dr.h.c. Klaus Volk, München; Dr. Joachim Walter, Adelsheim; Prof.Dr. Thomas Weigend, Köln; Prof.Dr. Peter Wetzels, Hamburg; Prof.Dr. Dieter Zimmermann, Darmstadt

\title{
Gegenreform erfasst Strafvollzugsgesetz
}

\section{Bemerkungen zur Situation des Strafvollzuges aus Anlass einer drohenden Verlagerung der gesetzgeberischen Zuständigkeit auf die Länder}

Gerhard Rehn er gegenwärtig Strafvollzug betreiben und gestalten will oder muss, der findet eine vergleichsweise komfortable Situation vor. Das gilt jedenfalls dann, wenn sich Politiker, Verwalter und Planer an empirisch gesicherten Kenntnissen über die Wirkung unterschiedlicher Reaktionen auf Straffälligkeit, an Erfahrungen mit daraus hergeleiteten rationalen Gestaltungsmaximen, am gedankenreichen Fundus der Strafvollzugsreformer, an den Grundsätzen des Strafvollzugsgesetzes (StVollzG) und an grundgesetzlich verankerten Menschenrechten und zivilen Standards orientieren. Tatsächlich setzt sich aber in die Nesseln, wer damit Ernst macht, denn unsere Zeit scheint davon nichts mehr wissen zu wollen. Nicht wenige Politiker gefallen sich zunehmend darin, mit populistischen Parolen die Gegenreform anzuheizen oder einfach nur auf der Welle des neuen (oder uralten?) Zeitgeistes mitzuschwimmen. Das ist gegenü- ber Betroffenen, den Tätern, ihren Angehörigen, (potentiellen) Opfern und der Gesellschaft insgesamt verantwortungslos, weil es um parteipolitischer Vorteile willen wider besseren Wissens geschieht, - um nur das Mindeste zu sagen. In einer solchen Situation wäre es verhängnisvoll, die Zuständigkeit für das StVollzG in die Hände der Länder zu legen, wie dies überraschend im Rahmen der Beratungen der Föderalismuskommission Ende des vergangenen Jahres geplant war. Wahrscheinlich würde die verbreitete faktische Missachtung seiner Inhalte alsbald normativ abgesegnet werden. Ein zweiter Wettlauf um den kürzesten Weg zurück zu einem scharfen Verwahrvollzug wäre zu befürchten.

\section{I.}

Tatsächlich zeichnet sich eine solche Entwicklung nur zu deutlich bereits $a b$. Sie kann recht präzise beschrieben werden. Die Trennlinie zwischen Reform und Reaktion wird überschritten, wenn der Straftäter als konkreter Mensch, als Rechtssubjekt und Mitglied der Gesellschaft aus dem Blick gerät und zum Objekt von Ausgrenzung und Vergeltung wird, auf dessen Kosten »ein Exempel $\mathrm{zu}$ statuieren « sei (Callies, 2001, S. 109). Sie wird überschritten, wenn dem Engagement der Strafrechtsreformer für »eine bessere Verwirklichung spezialpräventiver Ideen « (Kaiser \& Schöch, 2002, S. 231) der Rücken gekehrt wird und das Reaktionssystem nich länger allein darauf gerichtet ist, »die Fähigkeit des Verurteilten, sich in Freiheit ohne Straftat zu behaupten, auszubilden oder zu erhalten « (Stratenwerth, 1969, S. 46). Die Forderung, sich um ein sozial verantwortliches Leben $\mathrm{zu}$ mühen, schließt sodann aber auch ein, dass die sozialen Verhältnisse, vor allem im Strafvollzug, dies fördern, jedenfalls dazu nicht im Widerspruch stehen, damit, was vom Täter verlangt wird, ihm zugemutet und von ihm auch aufgegriffen werden kann (vgl. Baumann, 1969, S. 12).

Was hier nur angedeutet wird (ausführlicher Rehn, 2004, S. 526ff), entspricht dem Geist und den Grundsätzen des StVollzG. Man kann daher auch sagen, die Grenze von der Reform zur Restauration wird überschritten, sobald die Intentionen des Gesetzes, so unvollkommen es auch sein mag, nicht länger praxisleitende Richtschnur sind. Der gesetzte Rahmen ist längst nicht mit einem überzeugenden Bild gefüllt. Das liegt auch am StVollzG selbst, denn es ist zum Teil bis heute ein Ankündigungsgesetz geblieben, und viel zu wenig ist konkretisiert, was eingangs so großartig postuliert wird. Entsprechend unterschiedlich hat sich die Praxis in den Ländern entwickelt. Nahezu durchgängig zeigen sich große Mängel. Hier wäre bundesweit Reformbedarf im Normativen und Faktischen. Eine gemeinsame Anstrengung über Ländergrenzen und Parteien hinweg könnte dabei allein verhindern, dass weiterhin der Verlockung nachgegeben wird, komplexe Probleme auf sex \& crime populistisch zu verkürzen und aufschaukelnd konkurrierend parteipolitisch auszuschlachten. Davon sind wir aber weit entfernt. Doppelt weit nun, weil auch das Bewusstsein von einer Lücke zwischen Anspruch und Wirklichkeit, zwischen richtig und falsch man- 
cherorts zu schwinden scheint. So mag offenbar kaum ein mit Macht ausgestatteter Politiker das Wort führen, um auf der Grundlage rationaler Überlegungen z.B. für mehr Normalisierung im Strafvollzugsalltag, mehr offenen Vollzug und mehr Vollzugslockerungen zu plädieren.

\section{II.}

Eine grundlegende Quelle mehr oder minder virulenter Reformfeindlichkeit sind die in Bauwerken, Organisationsbräuchen, administrativen Vorschriften und in machen Köpfen verankerten Einstellungen und Traditionen über den »richtigen « Umgang mit Straftätern und darüber, wie sie angemessen zu bestrafen und zu verwahren seien. Die alten Traditionen begleiteten und behinderten die Reformarbeit der vergangenen Jahrzehnte, den Anspruch, Brücken zwischen dem Straftäter und der Gesellschaft zu erhalten, zu festigen oder überhaupt erst zu errichten. Getragen von einer positiven gesellschaftlichen Grundstimmung war dennoch vieles möglich. Gegenwärtig aber ist das alte, repressiv-ausgrenzende Denken recht verbreitet wieder dominant. Die Festigung der alten Strukturen wird oft scheinrational mit neuartigen Problemen gerechtfertigt, von denen bei Erlass des Gesetzes niemand habe wissen können: illegale Drogen, hoher Ausländeranteil, offene Grenzen, neue Formen der Gewaltkriminalität usw. Alles richtig, aber bei genauer Analyse kein Grund, die primär an einzelfallbezogener Eingliederungsabsicht orientierte Arbeit aufzugeben. Das StVollzG ist kein Schönwettergesetz, es erlaubt, ja fordert geradezu, im Vollzug intern zu differenzieren. Unter den vielen Gefangenen befindet sich seit jeher auch eine kleine Gruppe besonders gestörter und/oder hoch gefährdeter und gefährlicher Menschen. Darum müsse, hieß es z.B. schon bei Einsele, die Überleitung in Freiheit unter Berücksichtigung der teils ernsthaften Persönlichkeitsstörungen behutsam erfolgen (1970, S. 33). Auch die schon in den 1960er Jahren intensiv einsetzende Diskussion um die gesetzliche und inhaltliche Gestaltung der Sozialtherapie im Justizvollzug beschäftigte sich mit der Frage, wie mit besonders problematischen Gefangenen sinnvoll umzugehen sei.
Erinnert sei auch an die Phase des Terrorismus in den 1970er Jahren. So hat jede Zeit ihre besonderen Probleme und glaubt schnell, die jeweils schwierigste zu sein. Jedenfalls besteht aus der Sache heraus kein Anlass zu einem "Generalangriff « auf das Behandlungsziel des § 2 StVollzG (s. Dünkel, 2003). Gegenreformatorisch ist es auch, wenn gefordert wird, das Gesetz möge an die Folgen unterlassener Handlungen der Landesregierungen angepasst werden und z.B. die Erlaubnis zur gemeinschaftlichen Unterbringung von Gefangenen während der Ruhezeit festschreiben.

Die restaurative Entwicklung des Strafvollzuges wird am Beispiel des offenen Vollzuges und der Vollzugslockerungen besonders deutlich. Das Justizministerium in Hessen teilte schon 2002 mit, man habe seit 1998 die Zahl der Urlaubsgewährungen um gut zwei Drittel reduziert. In Hamburg wurde die Zahl der Ausgänge von 2001 auf 2004 (jeweils bis Oktober) um 35,8\%, die der Urlaube um 47,5\% verringert. Die niedersächsische Ministerin will nun ebenfalls die Haftbedingungen verschärfen. Neben härteren Sanktionen bei Drogenkonsum und selteneren Besuchsmöglichkeiten soll auch weniger »Freigang « gewährt werden (ZfStrVo, 2004， S. 357). Feest \& Lesting (2004) haben anhand aktueller Zahlen gezeigt, dass der »Wechsel des vollzugspolitischen Klimas « in »fast allen Bundesländern eine mehr oder weniger starke Rückentwicklung « der Lockerungen zur Folge hatte. Dieselbe Entwicklung zeigt sich in doppelter Hinsicht beim offenen Vollzug. Zum einen reduzieren insbesondere Hessen und Hamburg ihren entsprechenden Platzanteil deutlich; Hamburg von rund $23 \%$ aller Haftplätze auf zunächst (!) etwa $10 \%$. Der bundesweite Durchschnitt liegt bei 16,6\% (Bundeseinheitliche Strafvollzugsstatistik vom 31.12.2000, s. auch Dünkel, 2004, S.108ff). Zum anderen könnten Länder wie Thüringen, Bayern, Sachsen-Anhalt und Sachsen, deren Anteil an Plätzen des offenen Vollzuges unter $10 \%$ liegt, dadurch darin bestärkt werden, das Vorranggebot des $\S 10$ StVollzG weiterhin so eklatant zu missachten, so dass, vor allem auch vor dem Hintergrund verbreiteter Ausbaumaßnahmen im geschlosse- nen Vollzug, zumindest die relative Zahl der offenen Plätze - auf alles gesehen - deutlich rückläufig ist. Die Folgen sind verheerend und keineswegs nur punktueller Art. Sie strahlen auf den gesamten Vollzug aus und betreffen auch das Verhältnis zwischen Vollzug und Gesellschaft. So werden die bekannten gesetzlichen Lockerungszwecke unterlaufen, wonach der Gefangene seinen Angehörigen und dem Leben draußen möglichst wenig entfremdet werden soll, was im offenen Vollzug besser als im geschlossenen gelingt. Er soll, selbstverständlich unter Beachtung von Sicherheitsaspekten, möglichst frühzeitig in Lockerungen erprobt werden, um seine Fähigkeit zu einem sozial verantwortlichen Leben $\mathrm{zu}$ prüfen und an Mängeln arbeiten zu können. Und schließlich soll er noch aus dem Vollzug heraus in Arbeit integriert und mit einer angemessenen Unterkunft entlassen werden (vgl. Feest \& Lesting, 2005). Werden diese Prinzipien in immer mehr Fällen verlassen, dann verlieren Gefangene und die mit ihnen befassten Mitarbeiter eine Perspektive, auf die hin $\mathrm{zu}$ arbeiten sich lohnt. Für den Gefangenen ist es dann kaum noch möglich, die Berechtigung einer vorzeitigen Entlassung durch positive Leistungen $\mathrm{zu}$ belegen; die Entlassung erfolgt schließlich unvorbereitet ins Ungewisse. Während der Verbüßung ziehen sich die Gefangenen wieder mehr in sich und in die Subkultur der Insassen zurück. Vordergründige Anpassung, Lebensuntüchtigkeit und Aggressivität nehmen in den lebensfernen und inhumanen Strukturen des Verwahrvollzuges zu und belasten und gefährden nach der Entlassung Angehörige und Öffentlichkeit. Bei unkundigen Menschen wird durch diese Politik der institutionellen Aufrüstung das Bild des Rechtsbrechers mit negativen Emotionen und massenmedialen Verzerrungen in Übereinstimmung gebracht, woraus sich sodann scheinheilig weitere Legitimation für Repression beziehen lässt...

\section{III.}

Eingangs wurde gesagt, dass Strafvollzugsgestalter mehr denn je auf gesichertes Wissen zurückgreifen könnten. Einige wenige Fakten, die den gesetzlichen Vorrangs des offe- nen Vollzuges stützen, seien hier angeführt: Warum, ist zu fragen (zum Folgenden s. Dünkel, 2004; Kerner, 2004; Jehle, Heinz \& Sutterer, 2003, S. 37f; Schöch, 1996), warum nicht mehr Unterbringung im eingliederungsförderlichen und preiswerteren offenen Vollzug, wenn z.B. fast die Hälfte der inhaftierten Männer Freiheitsstrafen unter einem Jahr verbüßen, wenn rund $70 \%$ der Gefangenen nicht wegen eines Verbrechens gegen Leib und Leben verurteilt worden sind, wenn Vollzugslockerungen ganz überwiegend verantwortungsbewusst genutzt werden (der Missbrauch bezogen auf Gewährungen liegt unter einem Prozent, in wenigen Fällen kommt es zu neuen, meist geringen Straftaten), und schließlich, warum so massiv und zunehmend mehr geschlossener Vollzug mit seiner desozialisierenden Wirkung, wenn nur 29 \% der Entlassenen innerhalb von vier Jahren wegen eines neuen Deliktes in den Strafvollzug zurückkehren müssen? Aber auch Gefangene mit längeren Strafen brauchen, wenn sie nicht unvorbereitet auf die Strasse gestellt werden sollen, im Fortgang der Verbüßung die Überleitung durch offenen Vollzug oder, bei größerem Behandlungsbedarf, über eine sozialtherapeutische Einrichtung. Selbst wiederholt auffällige Täter seien, heißt es bei Kerner, »auf mittlere bis längere Sicht der Resozialisierung zugänglich« (2004, S. 32f).

Auch offener Vollzug ist in der Regel gegen spontanes Weglaufen gesichert, z.B. durch Fenstergitter, und er ist durch mit Sanktionen gestützte Hausordnungen geordnet. $\mathrm{Zu}$ seiner Fortentwicklung wäre es aber erforderlich, besondere für die große Zahl der lebensuntüchtigen und wiederholt mit (überwiegend) Bagatelldelinquenz auffälligen Männer ein intensives Trainingsprogramm an der Nahtstelle zwischen Drinnen und Draußen zu organisieren. Es wäre behördenübergreifend von externen und internen Kräften sowie Mitarbeitern freier Träger zu gestalten... Man möge sich, statt Destruktion zu betreiben, derartigen Projekten widmen und so dem allgemeinen Wohl dienen.

IV.

Anliegen der Strafrechts- und Strafvollzugsreform war (und ist) es, anstelle metaphysischer Ansprüche den konkreten Menschen mit seinen Ver- 
strickungen in den Blick zu nehmen (Schulz, 1973, S. 758). Auf sozial unverantwortliches Verhalten soll eher pragmatisch, von einem »sozial-rechtlichen Schuldbegriff « (Baumann, 1969, S. 12) ausgehend, so reagiert werden, dass über Lernvorgänge die Einübung in sozial verträgliches Handeln ohne Straftaten möglich wird. Unser Wissen über die Ursachen und den Verlauf abweichenden Verhaltens zeigt, dass dieses Programm in vielen Fällen erfolgreich ist und zum gesellschaftlichen Frieden beiträgt. Viele Bedienstete in den Anstalten setzen diese mühselige Arbeit auch unter widrigen Umständen fort, z.T. allein gelassen von der politischen Spitze. $\mathrm{Zu}$ den herkömmlichen, in den Ländern über viele Jahre hinweg verschuldeten Mängeln treten nun intensive restaurative Tendenzen. Sie behindern die Orientierung am einzelnen Menschen, weil sie ressortegoistisch allein auf eine risikolose VerbüBung setzen, sich um das Danach nicht scheren und den Täter erneut auf Eigenschaften reduzieren, die geeignet sind, eigene Einsperrungsund Strafbedürfnisse zu rechtfertigen (s. dazu Böllinger, 2004).

In einer solchen Situation ragt das StVollzG wie ein Relikt aus besonneneren Tagen in die Gegenwart. Es gibt zu dessen Zielen, Leitsätzen und zu einigen zentralen Einzelbestimmungen keine vernünftige Alternative. Sein Geist und seine Inhalte würden durch die Aufgabe der zentralen Zuständigkeit noch mehr verraten. Das Hamburger Beispiel zeigt dies exemplarisch besonders deutlich: Wer, wie Senator Kusch, den offenen Vollzug mehr als halbiert, Lockerungen annähernd halbiert, Haftbedingungen auch sonst weiter verschärft und eine funktionierende Sozialtherapie durch eine unsinnige und teure Verlagerung zerstört (um hier nur die wichtigsten Punkte $\mathrm{zu}$ nennen) und bei allem glauben macht, es werde dadurch mehr Sicherheit für Hamburg hergestellt, der verschleudert vollzugspolitisches Kapital und verschärft die Probleme (Rehn, 2003). Das haben dem Senator, dem Ersten Bürgermeister und den Bürgerschaftsfraktionen viele Wissenschaftler und Praktiker schriftlich gegeben. Was immer daraus entstehen mag: Das Strafvollzugsgesetz, das so sehr in das Leben von Menschen in extrem abhängiger Position eingreift, darf nicht in die Hände der Länder fallen und dort auf die schiefe Bahn wech- selnder und sachferner Interessen geraten. Politik ist manchmal nur halbstark, dort am meisten, wo sie besonders stark auftritt.

\section{Literatur:}

Baumann, J. (1969): Schuld und Sühne als Grundproblem heutiger Strafrechtspflege. In: J. Baumann (Hrsg.) (1969): Misslingt die Strafrechtsreform? Neuwied und Berlin: Luchterhand

Böllinger, L. (2004): Schuld-Strafe-Macht: Eine psychosoziale Rekonstruktion. In: G. Rehn, R. Nanninga \& A. Thiel (Hrsg.): Freiheit und Unfreiheit. Arbeit mit Straftätern innerhalb und außerhalb des Justizvollzuges. Herbolzheim: Centaurus, S. 53-77

Callies, R.-P. (2001): Die Strafzwecke und ihre Funktion. Straftheorie oder dialogische Strafrechtstheorie als Bezugsrahmen. In: G. Britz, H. Jung, H. Koriath \& E. Müller (Hrsg.): Grundfragen staatlichen Strafens. Festschrift für Heinz Müller-Dietz zum 70. Geburtstag, München: C. H. Beck, 99-118

Dünkel, F. (2003): Sicherheit als Vollzugsziel - Die Wende im Strafvollzug in Zeiten des Wahlkampfes: eine Initiative aus Hessen. In: Neue Kriminalpolitik 1/2003
Dünkel, F. (2004): Riskante Freiheiten? - Vollzugslockerungen zwischen Resozialisierung und Sicherheitsrisiko. In: G. Rehn, R. Nanninga \& A. Thie (Hrsg.): Freiheit und Unfreiheit. Arbeit mit Straftätern innerhalb und außerhalb des Justizvollzuges. Herbolzheim: Centaurus, S. $104-134$

Einsele, H. (1970). Referat. In: Ständige Deputation des Deutschen Juristentages (Hrsg.). Mit welchem Hauptinhalt empfiehlt es sich, ein Strafvollzugsgesetz zu erlassen? Verhandlungen des 48 Deutschen Juristentages 1070 in Mainz. München: Beck, S. 32-51.

Feest, J. \& W. Lesting (2004): Der Angriff auf die Lockerungen. Daten und Überlegungen zur Lockerungspolitik der Länder. In ZfStrVo (in Vorbereitung)

Jehle, J.-M., W. Heinz \& P. Sutterer (2003): Legalbewährung nach strafrechtlichen Sanktionen, Berlin: Bundesministerium der Justiz

Kaiser, G \& H. Schöch (2002): Strafvollzug. Eine Einführung in die Grundlagen. 5 Aufl. Karlsruhe: C.F.Müller

Kerner, H.-J. (2004): Freiheit und Unfreiheit. Zum Verlauf der Karrieren von Straftätern. In: G. Rehn, R. Nanninga $\&$ A. Thiel (Hrsg.): Freiheit und Unfreiheit. Arbeit mit Straftätern innerhalb und außerhalb des Justizvollzuges. Herbolzheim: Centaurus, $3-52$
Rehn, G. (2003): Ist eine rationale Strafvollzugspolitik heute noch möglich? Strafvollzug am Scheideweg. In: ZfStrVo, S. 70 - 76

Rehn, G. (2004): Reform und Gegenreform. Bemerkungen zur Situation und zu den Perspektiven des Strafvollzuges. In: G. Rehn, R. Nanninga \& A. Thiel (Hrsg.): Freiheit und Unfreiheit. Arbeit mit Straftätern innerhalb und außerhalb des Justizvollzuges. Herbolzheim: Centaurus, 523 -537

Schöch, H. (1996): Die Rechtswirklichkeit und präventive Effizienz strafrechtlicher Sanktionen. In: J.M. Jehle (Hrsg.): Kriminalprävention und Strafjustiz. Wiesbaden: Selbstverlag Kriminologische Zentralstelle, Bd. 17, S. 291-326

Schulz, W. (1972): Philosophie in der veränderten Welt. Pfullingen: Günther Neske

Stratenwerth, G. (1969): Schuld und Rechtfertigung. In: J. Baumann (Hrsg.) (1969): Misslingt die Strafrechtsreform? Neuwied und Berlin: Luchterhand

Dr. Gerhard Rehn, 1984 bis 1994 Leiter der Sozialtherapeutischen Anstalt Hamburg-Altengamme und bis 2000 Leiter der Abteilung Vollzugsgestaltung in der Justizbehörde Hamburg

\section{Strafvollzug ist Ländersache!? Was trotz bundeseinheitlicher Gesetzgebungs- kompetenz des Bundes möglich ist}

\section{- Dargestellt am Beispiel des offenen Vollzuges}

Frieder Dünkel

( ie negativen Folgen der von Gerhard Rehn (siehe nebenstehenden Bericht) thematisierten Verlagerung der Bundes- auf eine Landeskompetenz, wie sie die (insoweit glücklicherweise gescheiterte) sog. Föderalismuskommission vorgeschlagen hatte, können am nachfolgenden Beispiel des offenen Vollzugs eindrucksvoll belegt werden. Der offene Vollzug ist (ebenso wie andere Resozialisierungsmaßnahmen wie Vollzugslockerungen und Hafturlaub) zum Spielball restaurativer Vollzugspolitik geworden (vgl. schon Dünkel/Kunkat NK 2/1997). Das Strafvollzugsrecht betrifft einen Kernbereich staatlicher Tätigkeit, im Rahmen derer die intensivsten Eingriffe in die Rechte von verurteilten Bürgern stattfin- den. Der unsere Verfassung prägende Grundsatz, dass in den Ländern möglichst einheitliche Lebensverhältnisse herzustellen sind, muss im Strafvollzug in besonderem Maße Beachtung finden. Es war erklärtes Ziel der mehr als 100-jährigen Gesetzgebungsgeschichte bis zur Verabschiedung des Strafvollzugsgesetzes im Jahr 1976, der Auseinanderentwicklung der Lebensverhältnisse in den einzelnen Bundesländern entgegen $\mathrm{zu}$ wirken. Deshalb wurden $u$. a. zeitgleich mit dem StVollzG bundeseinheitliche Verwaltungsvorschriften erlassen.

Die nachfolgenden Daten verdeutlichen, dass schon der derzeitige Zustand des Strafvollzugs der Vorgabe einigermaßen vergleichbarer Le- bensverhältnisse und des unumstritten sinnvollen Ausbaus des offenen Vollzugs als besonders resozialisierungsfördernder Maßnahme immer weniger entsprochen wird. Allein seit 1996 hat sich der stichtagsbezogene Anteil von Gefangenen im offenen Vollzug in einigen Bundesländern drastisch verändert. Hamburg, ehemals liberale Hochburg eines resozialisierungs- und überleitungsorientierten Vollzugs, hat unter der konservativen Regierung und Justizsenator Kusch den Anteil von über $30 \%$ auf knapp 15\% mehr als halbiert. Hessen, ein Bundesland, das den offenen Vollzug als eines der ersten schon in den 1950er und 1960er Jahren modellhaft ausgebaut hatte, hat seine erfolgreiche Strategie (einschließlich der Direkteinweisung 